\title{
Gut bacterial communities in the freshwater snail Planorbella trivolvis and their modification by a non-herbivorous diet
}

\author{
Zongfu Hu ${ }^{1,2}$, Qing Tong ${ }^{2}$, Jie Chang ${ }^{1}$, Jianhua Yu ${ }^{1}$, Shuguo Li $^{1}$, Huaxin Niu ${ }^{\text {Corresp., }}{ }^{1}$, Deying Ma ${ }^{\text {Corresp. } 2}$ \\ ${ }^{1}$ College of Animal Science and Technology, Inner Mongolia University for Nationalities, Tongliao, People's Republic of China \\ 2 College of Animal Science and Technology, Northeast Agricultural University, Harbin, China \\ Corresponding Authors: Huaxin Niu, Deying Ma \\ Email address: niuhx@imun.edu.cn, 2967233453@qq.com
}

The freshwater pulmonate snail Planorbella trivolvis is a common species in various bodies of water but is not native to China. Planorbella trivolvis usually live on diets with high fibre content, such as water grasses, algae and fallen leaves. These snails can attach to the wall of a water tank or to water grass and can be transported overseas to China through the ornamental fish trade. There are few studies investigating the intestinal microbiota of freshwater snails. In this study, using culture-independent molecular analysis, we assessed for the first time the complexity of bacterial communities in the intestines of reared snails. The intestinal microbiota in the snails fed different diets, that is, herbivorous feed (HV) with high cellulose and non-herbivorous feed (NHV) with low cellulose, were analysed by Illumina sequencing. The results showed that the NHV-based diet significantly increased the body mass, shell diameter and specific growth rate of the snails after 60 days of rearing $(P<0.05)$. Histological experiments showed that the fat droplets in the epithelium columnar cells of the intestines of the NHV snails increased, and the cilia on these cells fell off. The sequencing results identified 486 and 195 OTUs in HV and NHV, respectively. Lots of bacteria were not reported previously in snails. The intestinal microbiota diversity index (Shannon, Simpson, Ace and Chao) in the NHV snails was significantly lower than that in the HV snails. The gut microbiota in the HV snails were predominantly Proteobacteria (52.97\%) and Bacteroidetes (28.75\%), while the gut microbiota in NHV snails were predominantly Proteobacteria (95.23\%). At the genus level, Cloacibacterium (24.60\%), Pseudomonas (4.47\%), OM6ON (6.12\%), and Rhodobacter (5.79\%) were observed to be abundant in HV snails. However, Aeromonas (85.4\%) was determined to be predominant in NHV snails. Functional prediction of the gut microbiome in snails by PICRUSt demonstrated a significant difference between the two groups, and the HV snails exhibited higher lignocellulose enzyme activity than did the NHV snails. This study represents a first step in characterizing the gut microbiota of the freshwater snail. Most of these microbes can process plant biomass and digest cellulose and lignocellulose, and the enzymes of these 
bacteria may have potential biotechnological applications in a variety of industrial processes. 
1 Gut bacterial communities in the freshwater snail Planorbella trivolvis and their

2 modification by a non-herbivorous diet

3 Zongfu $\mathrm{Hu}^{1,2}$ Qing Tong ${ }^{2}$ Jie Chang ${ }^{1}$ Jianhua $\mathrm{Yu}^{1}$ Shuguo $\mathrm{Li}^{1}$ Niu Huaxin ${ }^{1 *}$

4 Deying $\mathrm{Ma}^{2 *}$

5 1. College of Animal Science and Technology, Inner Mongolia University for

6 Nationalities, Tongliao City 028000;

7 2. College of Animal Science and Technology, Northeast Agricultural University,

8 Harbin city 150030

\section{ABSTRACT:}

The freshwater pulmonate snail Planorbella trivolvis is a common species in various bodies of water but is not native to China. Planorbella trivolvis usually live on diets with high fibre content, such as water grasses, algae and fallen leaves.

14 These snails can attach to the wall of a water tank or to water grass and can be 15 transported overseas to China through the ornamental fish trade. There are few 16 studies investigating the intestinal microbiota of freshwater snails. In this study,

17 using culture-independent molecular analysis, we assessed for the first time the

18 complexity of bacterial communities in the intestines of reared snails. The intestinal microbiota in the snails fed different diets, that is, herbivorous feed (HV)

20 with high cellulose and non-herbivorous feed (NHV) with low cellulose, were 
21 analysed by Illumina sequencing. The results showed that the NHV-based diet

22 significantly increased the body mass, shell diameter and specific growth rate of

23 the snails after 60 days of rearing $(P<0.05)$. Histological experiments showed that

24 the fat droplets in the epithelium columnar cells of the intestines of the NHV snails

25 increased, and the cilia on these cells fell off. The sequencing results identified 486

26 and 195 OTUs in HV and NHV, respectively. Lots of bacteria were not reported

27 previously in snails. The intestinal microbiota diversity index (Shannon, Simpson,

28 Ace and Chao) in the NHV snails was significantly lower than that in the HV

29 snails. The gut microbiota in the HV snails were predominantly Proteobacteria

$30(52.97 \%)$ and Bacteroidetes (28.75\%), while the gut microbiota in NHV snails

31 were predominantly Proteobacteria (95.23\%). At the genus level, Cloacibacterium

32 (24.60\%), Pseudomonas (4.47\%), OM6ON (6.12\%), and Rhodobacter (5.79\%)

33 were observed to be abundant in HV snails. However, Aeromonas $(85.4 \%)$ was

34 determined to be predominant in NHV snails. Functional prediction of the gut

35 microbiome in snails by PICRUSt demonstrated a significant difference between

36 the two groups, and the HV snails exhibited higher lignocellulose enzyme activity

37 than did the NHV snails. This study represents a first step in characterizing the gut

38 microbiota of the freshwater snail. Most of these microbes can process plant

39 biomass and digest cellulose and lignocellulose, and the enzymes of these bacteria 
40 may have potential biotechnological applications in a variety of industrial

41 processes.

42 Keywords: Planorbella trivolvis, freshwater snails, cellulose, gut microbiota,

43 functional prediction, intestine histology

45 INTRODUCTION

46 Planorbella trivolvis belongs to the family Plamorbidae and is primarily

47 distributed in Africa, Europe and North America. (Hunter, 1990). Planorbella

48 trivolvis is one of the most abundant taxa in the subclass Pulmonata, with more

49 than 250 species in 40 genera being recorded worldwide. These species can serve

50 as intermediate hosts for a variety of trematodes, such as Ribeiroia ondatrae and

51 echinostome, thereby contributing to the global disease burden (Klockars et al.,

52 2007; Peterson \& Nathan, 2007). Snails are common in ponds, lakes and marshes

53 and feed on algae, aquatic plants, the fallen leaves of terrestrial plants and various

54 types of detritus (Lombardo \& Cooke, 2002). Therefore, the ability of snails to

55 digest lignocellulose is notable, and they are thought to contain a gut microbiome

56 that is specialized in the rapid hydrolysis and fermentation of lignocellulosic plant

57 biomass (Pinheiro et al., 2015; Wijanarka, et al., 2016).

There is considerable bacterial diversity in the intestinal tracts of animals.

59 These bacteria can interact with host animals, enhance animal immunity, help to 
60 digest nutrients, and play important roles in inhibiting harmful foreign bacteria

61 (Lee et al., 2013; Carnevali et al, 2017; Guarner et al., 2003). Up to now, the gut

62 microbiota in herbivores have been well studied in many animals, such as cows,

63 ants, and pandas (Bergmann et al., 2015; Jami et al., 2013; Russell et al., 2009;

64 Tun et al., 2013).

65 However, the gut microbial communities of snails remain largely unstudied 66 (Joynson et al., 2017; Van Horn et al., 2012). The early study of intestinal

67 microbiota in snails employed culture-based methods, and a limited number of

68 bacteria were identified, such as Enterobacter, Enterococcus, Lactococcus, and

69 Clostridium (Charrier et al., 2006). Next, molecular biology techniques were used

70 to study the snail gut microbiota, such as DGGE fingerprint analysis, which has

71 been utilized to obtain the structural composition of the dominant bacterial

72 community in the snail Helix pomatia, and the number of identified bacteria was

73 also limited (Nicolai et al., 2015). Recently, high-throughput sequencing based on

74 parts of 16S rRNA gene, which can reveal more kinds of microbiota even that with

75 low abundance, were used to study the gut microbiomes in freshwater snails

76 Biomphalaria pfeifferi, Bulinus africanus, Helisoma duryi and Radix ovata (Van

77 Horn et al., 2012; Hu et al., 2018). Furthermore, the intestinal microorganisms of

78 snail play an important role in cellulose decomposition. Some studies have isolated

79 cellulolytic bacteria from the intestine of snails by carboxymethylcellulose (CMC) 
80 plates (Charrier et al., 2006; Pinheiro et al., 2015). Other studies, using

81 metagenomic, have identified a great number of genes involved in lignocellulosic

82 breakdown in intestinal microorganisms of snails Achatina fulica, Arion ater

83 (Cardoso et al. 2012; Joynson et al., 2017).

84 The main focus of this work was to characterize intestinal microbiomes of

85 Planorbella trivolvis shaped by diets. Previous studies showed that high-fibre or

86 low-fibre diets shape the intestinal microbiota and microbial metabolites and affect

87 the growth performance and intestinal morphology in various animals, such as pigs

88 and rats (Stark et al., 1996; Heinritz et al., 2016; Coble et al., 2018). Therefore, the

89 variation in fibre content in diets may affect the intestinal microbiota in snails or

90 affect the growth and intestinal status of snails. However, the effects of specific

91 dietary modulation on the intestinal microbiota and growth of snail have not been

92 fully elucidated.

93 In this study, a culture-independent molecular analysis based on the 16S rRNA

94 gene was performed (1) to demonstrate the effect of the diet (high-cellulose and 95 low-cellulose) on gut microbiota in Planorbella trivolvis and (2) to examine 96 functional differences in the microbiomes of snails fed different diets. The effects 97 of diet on snail growth and intestinal morphology were also examined in this study. 
100 Rearing of snails

101 The snails were originally purchased from the ornamental fish market, and a

102 breeding colony were formed in the laboratory. When a sufficient number of

103 newborn snails were reared to about 5-7 $\mathrm{mm}$ of shell diameter, a total of 120

104 juvenile snails with uniform size $(5.88 \pm 0.14 \mathrm{~mm}$ of shell diameter $)$ were selected

105 and randomly distributed into two groups by their diets: herbivorous groups (HV)

106 (60 snails) and non-herbivorous groups (NHV) (60 snails). Each group had three

107 replicate aquariums with 20 snails in each repetition. HV group was fed dry alfalfa

108 contain a high content of cellulose (26\%), and NHV group was fed market-

109 purchased pellet feed (Takara sakana-ii, for ornamental fish feeding), which

110 contained low cellulose $(5 \%)$ (Table 1$)$. The aquaculture water temperature was

111 maintained at $24 \sim 25{ }^{\circ} \mathrm{C}$, with a pH of $6.7 \pm 0.4$. The snails were reared for 60

112 days and no snails died during rearing.

113 Sample collection and processing

114 After 60 days of rearing, the body length and weight of snails were

115 determined first. Next, the intestines were sampled. When the intestines were

116 collected, the snails were cleaned with tap water, and the surface of the shell was

117 subsequently wiped with $70 \%$ alcohol. The shells were carefully broken and

118 disassembled from the snail body. After that step, the intestines were extracted

119 carefully to avoid rupturing the gut wall. Next, the intestines were sampled and 
120 contained their contents. All dissections were conducted under aseptic conditions.

121 For histological examination, the collected intestines were fixed in $4 \%$

122 formaldehyde for histological examination. Because the intestines of individual

123 snails were too small to meet the sampling requirements, the intestines and

124 contents of the 5-8 snails were packed into a centrifuge tube to form a repeat

125 sample for sequencing.

\section{Growth index}

127 The growth performance of snails after 60 days of rearing was characterized

128 by their weight gain rate (WGR) and specific growth rate (SGR). The WGR was

129 calculated by comparing their weight gain (WG) with their initial average weight

130 (IW). The SGRs were obtained by dividing their weight gain by 60 days. The

131 formulas of WGR and SGR are as follows:

132

133

$\operatorname{SGR}(\% / \mathrm{d})=[(\mathrm{TW}-\mathrm{IW}) / \mathrm{d}] \times 100$

134

WGR: Weight gain rate; SGR: Specific growth rate; TW: terminal weight; IW:

135 initial weight; d: rearing days

\section{Intestinal morphology analysis}

137 To examine the effect of diets on the intestinal morphology of snails, the intestinal

138 samples were washed in saline solution and fixed in 4\% paraformaldehyde solution 
139 above $48 \mathrm{~h}$. Next, the fixed samples were successively treated as follows:

140 dehydrated by an alcohol gradient $(50 \%, 70 \%, 90 \%$ and $100 \%)$, xylene

141 transparency, and paraffin embedding. Three paraffin sections from each sample

142 were cut and stained by the haematoxylin-eosin (HE) method. Last, the stained

143 slice was sealed by neutral gum and subjected to observation and scenery shooting

144 using a panoramic scanner (3DHISTECH, Pannoramic MIDI, Hungary).

145 Determination of intestinal microbiota diversity

146 The intestine samples obtained as described above contained the contents used for

147 genome extraction. Total DNA was extracted using the FAST DNA ${ }^{\mathrm{TM}}$ Spin Kit for

148 soil (MP-BIO, Santa Ana, CA, USA) following the protocol of the manufacturer.

149 The extracted DNA was subjected to $1.5 \%$ agarose-gel electrophoresis, and the

150 DNA concentration and OD260 nm/OD280 nm (OD, optical density) value were

151 determined with a Nanodrop spectrophotometer (Thermo Scientific, Wilmington, 152 USA).

153 To detect intestinal bacteria, the V3 V4 regions of the bacterial 16S rRNA 154 gene were amplified with DNA primers 338F $\quad\left(5^{\prime}\right.$ 155 ACTCCTACGGGAGGCAGCAG-3') and ${ }^{\prime}$ (5'156 GGACTACHVGGGTWTCTAAT-3') (Mori et al., 2013). The 12 bp barcodes on

157 both ends of the primers were used to identify the sequences of different samples.

158 Next, PCR amplification was conducted, and the procedure was as follows: initial 
159 denaturation at $95^{\circ} \mathrm{C}$ for 3 min followed by 27 cycles of denaturing at $95^{\circ} \mathrm{C}$ for 30

$160 \mathrm{~s}$, annealing at $55^{\circ} \mathrm{C}$ for $30 \mathrm{~s}$ and extension at $72{ }^{\circ} \mathrm{C}$ for $45 \mathrm{~s}$, single extension at

$16172{ }^{\circ} \mathrm{C}$ for $10 \mathrm{~min}$, and ending at $4{ }^{\circ} \mathrm{C}$. The amplified products were subjected to

162 electrophoresis and extracted from a 2\% agarose gel. Next, the products were

163 purified using the AxyPrep DNA Gel Extraction Kit (Axygen Biosciences, Union

164 City, CA, USA). After Qubit quantification and detection, amplicons from each

165 PCR sample were normalized to equimolar amounts, and high-throughput

166 sequencing was performed using the $2 \times 300 \mathrm{bp}$ protocol on an Illumina MiSeq

167 PE300 platform (Illumina, San Diego, CA, USA).

168 The raw reads were deposited into the NCBI Sequence Read Archive (SRA)

169 database (Accession Number: PRJNA640745).

170 Raw fastq files were demultiplexed, quality-filtered by Trimmomatic (Bolger

171 et al., 2014) and merged by FLASH. UPARSE (Edgar et al., 2013) was utilized to

172 conduct operational taxonomic unit (OTU) clustering analysis at 97\% identity, and

173 chimaeric sequences were identified and removed using UCHIME (Edgar et al.,

174 2011). Next, the representative OTUs were analysed on the Qiime platform

175 (Caporaso et al., 2010) against the Silva_132 16S Database (http://www.arb-

176 silva.de) to determine taxonomy.

177 The heatmap analysis was performed in the heatmap package in R. Venn and

178 PCoA analyses were performed in the vegan package in $\mathrm{R}$. The alpha -diversity of 
179 the gut bacterial community, containing indices of Sobs, Shannon, Chao, Simpson, 180 Ace, and coverage, was analysed by Mothur (Schloss et al., 2009). Biomarker 181 discovery was performed on the linear discriminant analysis (LDA) effect size 182 (LEfSe) to identify the specific organisms whose relative abundances differ 183 between two groups of samples (Segata et al., 2011).

184 To examine functional differences in the microbiome of snails, the functional 185 attributes of metabolic genes from the snail gut microbiota (KEGG Orthologs, KOs) 186 were predicted by a phylogenetic investigation of communities by reconstruction 187 of unobserved states (PICRUSt), which were obtained by macthing the sequencing 188 data against the genomic KEGG database (Langille et al., 2013). As a 189 computational approach, PICRUSt can predict the functional composition of an 190 obtained metagenome using marker gene data and a database of reference genomes 191 (Langille et al., 2013).

192

193

194 growth data of snails. $P$ values $<0.05$ were considered to indicate significant 195 196

\section{RESULTS}


199 The initial size and weight did not differ between the two groups $(P>0.05)$ (Table

200 2). After 60 days of rearing, the diameter of the final shell of the HV snails was

201 about $10.53 \mathrm{~mm}$, representing an increase of $176.08 \%$ compared with the initial

202 shell diameter (Table 2). The final shell diameter of NHV snails was about 11.86

$203 \mathrm{~mm}$, representing an increase of $205.19 \%$ compared with the initial shell diameter;

204 thus, and the final shell diameter of snails in the NHV group was significantly

205 larger than that of the HV group $(P<0.05)$. The endpoint weight of HV group was

$2060.219 \mathrm{~g}$, and that of NHV group was $0.432 \mathrm{~g}$. The weight gain rate of the NHV

207 group (566.90\%) was significantly higher than that of the HV group (321.40\%) $(P$

$208<0.05)$. Also, the specific growth rate of snails in the NHV group $(3.16 \%)$ was

209 significantly higher than that in the HV group $(1.94 \%)(P<0.05)$ (Table 2$)$.

\section{Intestinal morphology}

211 To examine the effect of feed on the intestinal morphology of snails, histological

212 examination were conducted (Figure 1). The differences between treatment groups

213 were clearly shown in the simple columnar epithelium cells (SCEC). For the HV

214 snails, the intestinal structure was intact, and the SCEC arrangement was regulated.

215 The microvilli were tightly attached to the SCEC. For the NHV snail, the intestinal

216 SCECs were filled with fat droplets and thus appear transparent in this image

217 (Figure 1C, D). The fat droplets occupied the cytoplasm of the SCEC, and the

218 nucleus was observed to be indented (Figure 1C, D), indicating that low fibre 
219 feeding leads to increased fat deposition in intestinal epithelial cells. The microvilli

220 were detached to a certain extent from the SCEC.

\section{Alpha diversity based on 16S rRNA gene sequencing}

222 A total of 737,284 raw reads were generated using the Illumina MiSeq sequence

223 platform and 485,342 high quality sequences were obtained (following quality

224 control and sequence filtration) with an average length of $459.9 \mathrm{bp}$. The mean

225 number of sequences per sample was 54,277.60 $\pm 6,298.01$ in HV snails and

$22639,606.60 \pm 6,846.06$ in NHV snails (Table 3). The 31,486 rarefied sequences

227 were clustered into 525 OTUs at 97\% identity, with 486 and 195 OTUs being

228 identified in HV and NHV, respectively.

229 The obtained 525 OTUs include members of 419 species, 304 genera, 184

230 families, 108 orders, 37 classes and 19 phyla. There were 18 phyla, 286 genera,

231108 orders, 36 classes, 486 OTUs belonging to HV, and there were 13 phyla, 52

232 orders, 21 classes, 132 genera, and 195 OTUs belonging to NHV. All of the alpha-

233 diversity indices (Sobs, Shannon, Simpson, Ace, Chao) were significantly different

234 between HV and NHV populations $(P<0.05)$ (student's t-test) (Table 3$)$. The

235 Good's coverage for the observed OTUs was above $99.70 \%$, and the rarefaction

236 curves showed clear asymptotes (Fig. 2A), which, taken together indicated that the

237 given level of sampling effort was sufficient to capture the bacterial communities

238 in snails. Notably, there were clear differences for the rarefaction and rank- 
239 abundance curves between the treatment groups (Fig. 2A, 2B). The abundance

240 analysis showed that there were only 5 OTUs in the NHV snails with abundances

241 greater than 1\%, while there were as many as 22 OTUs in the HV snails (Fig. 2B).

242 The total abundance of OTUs with abundance greater than $1 \%$ reached $79.68 \%$ in

243 the HV snails and $83.39 \%$ in the NHV snails. The number of core OTUs identified

244 in all samples was 22 (Fig. 2C).

245 Venn analysis showed that the bacteria belonging to 12 phyla, 77 families, and

246114 genera were shared by two groups: 6 phyla, 97 families, and 172 genera were

247 unique in HV snails, and 1 phylum, 10 families, and 18 genera were unique in

248 NHV snails (Fig. 2D-F).

\section{Composition of snail intestinal microbiota}

250 The phyla Proteobacteria (52.97\%) and Bacteroidetes $(28.75 \%)$ were predominant

251 among the bacteria in the HV group (Fig. 3A). The phyla with abundances above 1\%

252 were Actinobacteria, Verrucomicrobia, Chloroflexi, Cyanobacteria, and

253 Chlamydiae. However, only Proteobacteria (95.23\%) was predominant among the

254 bacteria in NHV snails. Bacteroidetes was the other phylum with an abundance 255 above $1 \%$.

256 At the family level, there were 20 families and 8 families with abundances

257 above $1 \%$ in the HV and NHV snails, respectively. Weeksellaceae (24.6\%) and 
258 Rhodobacteraceae (12.38\%) showed high abundance in HV snails.

259 Aeromonadaceae (85.40\%) dominated the microbiota in NHV snails (Fig. 3B).

260 At the genus level, the composition of intestinal microbiota differed between the

261 HV group and the NHV group (Fig. 3C). In the HV group, there were 22 genera

262 with abundances above 1\%. Cloacibacterium (24.60\%) showed the highest

263 abundance in the HV groups. Others, such as Pseudomonas (4.47\%), OM6ON

264 (6.12\%), and Rhodobacter (3.78\%) were also abundant. In the NHV snail, there

265 were 7 bacteria with abundances higher than 1\%. Aeromonas, exhibited the highest

266 abundance, accounting for $85.40 \%$.

267 The heat map analysis constructed from the top 35 abundant genera, reflected

268 that the intestinal microbiota in the two groups was different (Fig. 4). The

269 Wilcoxon rank-sum test showed that the abundance of 26 genera was significantly

270 higher in HV snails than in NHV snails $(P<0.05)$. Only two genera (Aeromonas

271 and Comamonas) with significantly higher abundances were observed in NHV

272 snails.

\section{Clustering of the snail gut bacterial community}

274 Clustering analysis of intestinal microbiota associated with diet were performed.

275 Principal coordinates analysis (PCoA) of sequencing data using pairwise weighted

276 and unweighted UniFrac distances showed that the bacterial community structure

277 of HV was different from that of NHV (Fig. 5). UniFrac clearly separated different 
278 microbiota efficiently by diet, demonstrating the importance of food as a driver of

279 community composition in this freshwater snail.

280 LEfSe analysis (the LDA threshold was 4) was used to screen out

281 microorganisms with significant differences in the snail intestines of the two

282 groups fed different diets. There were 11 bacterial genera that were significantly

283 enriched in the intestinal samples of the HV group, and there was one genus

284 (Aeomonas) that was enriched in the NHV group (Fig. S1). When the LDA

285 threshold was set to 2, 114 bacterial genera were significantly distributed in the

286 intestinal samples of the HV group, while only 4 bacterial genera were

287 significantly distributed in the NHV group (Table S1).

288 Although the intestinal microbiota of snails was influenced by food type, we 289 found that 27 genera existed as core microbiota of all samples in both groups.

290 Among these genera, 19 were affiliated with Proteobacteria, 4 were affiliated with

291 Bacteroidetes, 3 were affiliated with Actinobacteria, 2 were affiliated with

292 Verrucomicrobia and 1 was affiliated with Chloroflexi (Table 4).

293 Predictive Metagenomic Profiling

294 PICRUSt analysis was performed to generate a predictive functional profile to gain 295 insight into the metabolic capacity of the enteric microbiome (Fig. 6). Based on 296 this analysis, the relative abundance of several COG functions was significantly 297 different between feeding regimens, such as the enrichment of genes involved in 
298 energy production and conversion, lipid transport and metabolism in HV, and the

299 enrichment of genes involved in amino acid transport and metabolism, signal

300 transduction mechanisms in NHV. No significant differences were observed for the

301 relative abundance of genes involved in carbohydrate transport and metabolism,

302 inorganic ion transport and metabolism, and transcription.

303 Because plant fibre degradation requires a diverse suite of enzymes for 304 complete hydrolysis, the abundance of genes for several lignocellulose-active 305 enzymes was also examined (Fig. 7). Eighteen enzymes determined to be more 306 enriched in HV snails than in NHV snails, acting upon cellulose, hemicellulose,

307 lignin, and cello-oligosaccharides, respectively. Six enzymes were more enriched 308 in NHV than HV snails, acting upon hemicellulose, and cello-oligosaccharides, 309 respectively (Fig. 7). Enzymes related to protein degradation were also analyzed. 310 The result showed that a large number of genes related to aminopeptidase, 311 dipeptidase, carboxypeptidase and endopeptidase were more enriched in HV snails 312 than in NHV snails (Fig. 8).

314 DISCUSSION

315 For invertebrates, the study of gut microbes remains an open question and warrants 316 further research to characterize roles played by bacteria. In this work, we 317 investigated the effect of diets, containing a high (herbivorous diets, HV) or low 
318 (non-herbivorous diets, NHV) cellulose, on gut bacterial community structure in

319 Planorbella trivolvis. We also compared the effect of these diets on the growth and

320 gut histological morphology. NHV significantly promoted the body length and

321 body weight growth of snails, indicating that snails obtained more crude protein

322 and energy and less crude fibre when feeding on pellet feed. However, for

323 herbivorous feed, namely, alfalfa, the snails needs to use their radulas to scrape the

324 plant ingredients; therefore, the feeding efficiency is considerably lower.

325 The two diets were observed to influence the intestinal morphology of snails.

326 As the results showed, there were no fat droplets in the simple columnar

327 epithelium cells (SCECs) of HV snails. While in NHV snails, the intestinal SCECs

328 are filled with fat droplets. The reason for this phenomenon may lie in diets in

329 NHV with low dietary fibre, which contain more soluble carbohydrates and would

330 be easily digested and absorbed by snails. Furthermore, dietary soluble

331 carbohydrates can promote intestinal lipid accumulation in aquatic organisms

332 (Zhao et al., 2020; Castro et al., 2019). Therefore, the fat droplets occupied the

333 cytoplasm of the SCECs, and the nucleus was indented, which may be related to a

334 disturbed state of the normal metabolism of the cell and caused the detachment of

335 microvilli from SCEC in NHV snails.

336 Bacteria in the digestive tract may have the ability to ferment cellulose and

337 chitin to produce substances that snails can easily absorb, such as small molecules 
338 of organic acids and sugars (Speiser, 2001; Charrier et al., 2006). In this study, the

339 bacterial community in HV snails was dominated by Proteobacteria, Bacteroidetes

340 and Actinobacteria, indicating which bacteria may be related to plant digestion. In

341 fact, previous studies on herbivores have observed that Proteobacteria are the

342 dominant species in snails (Cardoso et al., 2012; Joynson et al., 2017; Nicolai et

343 al., 2015). In this study, we found that Bacteroidetes and Actinobacteria were also

344 associated with plant-eating gastropods. At the phylum level, the research results

345 of Van Horn et al. (2012) on the three Planorbidae have some similarities with ours.

346 Their study showed that Proteobacteria, Bacteroidetes, and Acidobacteria were

347 present and dominant in the intestines of these snails. A large number of bacterial

348 species belonging to Proteobacteria, Bacteroidetes, and Acidobacteria are also

349 observed in the gut of termites, which eat lignocellulose entirely (Mikaelyan et al.,

350 2017). However, for the American bison, a herbivore, the gut microbiota was very

351 different from that of the freshwater gastropods, with the order of abundance from

352 most to least being Firmicutes (53\%), Bacteroidetes (33\%) and Tenericutes (4\%)

353 (Bergmann et al., 2015).

354 Core microbes are microbes that are consistently present in a particular habitat,

355 such as intestines of animals, despite the high variable conditions in that habitat. In

356 this study, 27 genera were observed as core microbiota between the two dietary

357 groups, among which Cloacibacteria, Aeromonas and Pseudomonas had the 
358 abundance above $1 \%$ in both groups (HV and NHV), and Aeromonas,

359 Pseudomonas, Flavobacterium, and Enterobacteriaceae families have been

360 characterized as common in terrestrial or aquatic gastropods by previous studies

361 (Cardoso et al., 2012; Joynson et al., 2017). Although the role of the core

362 microbiota in the gut microbiota of animals is not well understood, it is likely

363 critical and needs further study (Shade and Handelsman, 2012; Kokou et al.,2019).

364 Some bacteria can be found both in the gut microbiota of snails and free-living

365 in the natural water environment. Cloacibacterium, which is abundant in HV snails,

366 is a facultative anaerobic and gram-positive bacterium and can be isolated from

367 freshwater sediments (Allen et al., 2016). Rhodobacter and Pseudomonas, widely

368 distributed in seawater and fresh water, were also found in the intestinal microbiota

369 of Potamopyrgus antipodarum, a freshwater snail from New Zealand (Vesbach et

370 al., 2016). Vesbach et al. (2016) determined that Rhodobacter colonized the

371 intestinal tract of Potamopyrgus antipodarum and formed symbionts with the host.

372 Symbiosis of Rhodobacter with host was also observed in sponges (Halichondria

373 Panicea) and Daphnia (Althoff et al., 1998; Qi et al., 2009).

374 There were 26 genera significantly increased in HV snails compared to NHV

375 snails, which were potentially plant-related intestinal microbiota, such as

376 Cloacibacteria, Pseudomonas, Flavobacterium, Mycobacterium, and Rhodobacter.

377 Pseudomonas has been identified as a cellulolytic species in invertebrates (Huang 
378 et al., 2012). In fact, most plant-related bacteria varied with the snail species.

379 Cardoso et al. (2012) reported that the bacterial taxa closely related to herbivores

380 are Pseudomonas, Clostridiaceae, Lactococcus, Bacteroides, Flavobacteriaceae,

381 Mucilaginibacter, Citrobacter, Klebsiella, Aeromonas, Acinetobacter, and

382 Comamonas. Among these bacteria, Pseudomonas, Flavobacteriaceae and 383 Aeromonas were found in our study. Microbacterium, Cellulosimicrobium, 384 Nocardiopsis, Aeromonas, Flavobacterium, Klebsiella were isolated from the 385 intestines of Achatina fulica with cellulose degradation activity (Pinheiro et al. 386 2015), and in which only Aeromonas and Flavobacterium were found in our study.

387 In this study, the intestinal microbiota of freshwater snails was modified by 388 diet, which was also reported in other animals, such as mammals (Muegge et al., 389 2011) and fish (Kokou et al., 2019). A similar study reported that the sugarcane390 based diet altered the gut microbiota of snail Achatina fulica (Cardoso et al., 2012).

391 Although Aeromonas was abundant (exhibiting an abundance of 85.40\%) in NHV

392 and was scarce in HV (1.78\%), this result does not mean that Aeromonas was 393 incapable of digesting plant fibre. Pinheiro et al. (2015) found that the 6 394 Aeromonas isolated from snail Achatina fulica all had the ability to hydrolyse 395 lignocellulose. Similar conditions were found in the genus Comamonas, which also 396 exhibited significantly higher abundance in NHV than in HV snails, but relevant 
397 studies also indicate that it may be related to herbivorous species (Cardoso et al., 398 2012).

399 However, more species in NHV snails (154 genera) disappeared or decreased 400 their abundance significantly compared to HV snails, indicating that these species 401 were better adapted to the intestinal environment of herbivorous diet than the 402 intestinal environment of non-herbivorous diet. Little is known about the role of 403 this microbiota in, for instance, assisting the host with food digestion or immunity. 404 The variation of these gut bacteria will provide better insights into the interaction 405 of snails and their gut microbiota.

406 PICRUSt analysis showed that different feed treatments had a significant 407 effect on the function of snail intestinal microbiota. Most COG functions had 408 significant differences between the HV and the NHV snails. Further KO analysis 409 showed a significant difference in the gene abundance of lignocellulose-active 410 enzymes between the two groups. The abundance of these lignocellulose-related 411 genes was mostly higher in the HV group than in the NHV group, indicating that 412 the intestinal microbiota in herbivorous snails were more effective in at 413 lignocellulosic metabolism than those in non-herbivorous snails. A large number of 414 genes related to protein degradation were higher in HV than in NHV snails, 415 indicating a more efficient protein utilization in herbivorous feeding snails, and 416 this will be helpful to develop microbial proteases from animal intestine. 


\section{CONCLUSIONS}

419 Our study provides the first characterization of gut community diversity of 420 Planorbella trivolvis, providing insight into gut community structure within 421 freshwater snails. Compared with herbivorous feeding (HV), although non422 herbivorous feeding (NHV) promoted the growth of snails, it caused an 423 accumulation of lipid droplets in intestinal mucosal cells. A set of 22 core bacteria 424 was determined to be consistently associated in relatively high abundance $(>1 \%)$ 425 with the diversity of Planorbella trivolvis. Compared with herbivorous feeding, 426 non-herbivorous feeding reduced the alpha-diversity of the intestinal microbiota of 427 snails and changed the composition of intestinal bacterial communities. For 428 example, the abundance of many bacteria decreased or disappeared. Functional 429 prediction showed that the abundance of cellulose degradation genes in the 430 intestinal microbiota of HV snails was considerably higher than that of NHV snails.

431 In summary, our findings showed novel snail-microbe associations and, 432 furthermore, suggest that the variety of bacteria within the gut might promote a 433 better digestion ability of the host to different diets. 
436 Allen TD, Lawson PA, Collins MD, Falsen E, Tanner RS. 2006.

437

438

439

440

441

442

443

444

445

446

447

448

449

450

451

452

453

454

Cloacibacterium normanensegen. nov., sp. nov., a novel bacterium in the family Flavobacteriaceae isolated from municipal waste water. International Journal of Systematic Evololutionary Microbiology 56(6):1311-1316 DOI 10.1099/ijs.0.64218-0

Althoff K, Schütt C, Steffen R, Batel R, Müller WEG. 1998. Evidence for a symbiosis between bacteria of the genus Rhodobacter and the marine sponge Halichondria panicea: harbor also for putatively toxic bacteria? Marine Biology 130: 529-536 DOI 10.1007/s002270050273

Bergmann GT, Craine JM, Robeson MS, II, Fierer N. 2015. Seasonal shifts in diet and gut microbiota of the American Bison (Bison bison). PLOS ONE 10(11): e0142409 DOI 10.1371/journal. pone.0142409

Bolger AM, Lohse M, Usadel B. 2014. Trimmomatic: a flexible Trimmer for Illumina sequence data. Bioinformatics $\quad 30: \quad 2114-2120$. DOI 10.1093/bioinformatics/btu170

Cardoso AM, Janaína JV, Cavalcante JJV, Cantão ME, Thompson CE, Flatschart RB, Glogauer A, Scapin SMN, Sade YB, Beltrão PJMSI, Gerber AL, Martins OB, Garcia ES, de Souza W, Vasconcelos ATR. 2012. Metagenomic analysis of the microbiota from the crop of an invasive snail 
reveals a rich reservoir of novel genes. PLOS ONE 7(11): e48505 DOI 10.1371/journal.pone.0048505

457

Caporaso JG, Kuczynski J, Stombaugh J, Bittinger K, Bushman FD, Costello EK. Fierer N, Pena AG, Goodrich JK, Gordon JI, Huttley GA, Kelley ST, Knights D, Koenig JE, Ley RE, Lozupone CA, McDonald D, Muegge BD, Pirrung M, Reeder J, Sevinsky JR, Peter JT, Walters WA, Widmann J, Yatsunenko T, Zaneveld J, Knight R. 2010. QIIME allows analysis of highthroughput community sequencing data. Nature Methods 7: 335-336 DOI 10.1038/nmeth.f.303

Carnevali O, Maradonna F, Gioacchini G. 2017. Integrated control of fish metabolism, wellbeing and reproduction: the role of probiotic. Aquaculture 472: 144-155 DOI 10.1016/j.aquaculture.2016.03.037

Castro C, Couto A, Diógenes AF, Corraze G, Panserat S, Serra CR, OlivaTeles A. 2019. Vegetable oil and carbohydrate-rich diets marginally affected intestine histomorphology, digestive enzymes activities, and gut microbiota of gilthead sea bream juveniles. Fish Physiology and Biochemistry 45(2): 681695. DOI 10.1007/s10695-018-0579-9

Charrier M, Fonty G, Gaillard-MartinIe B, Ainouche K, and Andant G. 2006. Isolation and characterization of cultivable fermentative bacteria from the intestine of two edible snails, Helix pomatia and Cornuaspersum (gastropoda: 
475

476

477

478

479

480

481

482

483

484

485

486

487

488

489

490

491

492

493

494

pulmonata). Biological Research 39(4): 669-681 DOI 10.4067/S0716-

\section{0}

Coble KF, Derouchey JM, Tokach MD, Dritz SS, Goodband RD, Woodworth JC. 2018. Effects of withdrawing high-fiber ingredients before marketing on finishing pig growth performance, carcass characteristics, and intestinal weights. Journal of Animal Science 96(1): 168-180 DOI 10.1093/jas/skx048

Edgar RC, Haas BJ, Clemente JC, Christopher Q, Rob K. 2011. UCHIME improves sensitivity and speed of chimera detection. Bioinformatics. 27, 21942200 DOI 10.1093/bioinformatics/btr381

Edgar R. 2013. UPARSE: highly accurate OTU sequences from microbial amplicon reads. Nature Methods 10: 996-998 DOI 10.1038/nmeth.2604

Guarner F, Malagelada JR. 2003. Gut flora in health and disease. The Lancet 361(9356):512-519 DOI 10.1016/S0140-6736(03)12489-0

Heinritz SN, Weiss E, Eklund M, Aumiller T,Louis S, Rings A, Messner S, Camarinha-Silva A, Seifert J, Bischoff SC, Mosenthin R. 2016. Intestinal microbiota and microbial metabolites are changed in a pig model fed a highfat/low-fiber or a low-fat/high-fiber diet. PLoS One 11(4): e0154329 DOI 10.1371/journal.pone.0154329

Huang S, Sheng P, Zhang H. 2012. Isolation and identification of cellulolytic bacteria from the gut of Holotrichia parallela larvae (Coleoptera: 
497 Hu Z, Chen X, Chang J, Yu J, Niu H. 2018. Compositional and predicted 498 functional analysis of the gut microbiota of Radix auricularia (Linnaeus) via 499 high-throughput Illumina sequencing. PeerJ 6: e5537 DOI 10.7717/peerj.5537 501

Jami E, Israel A, Kotser A, Mizrahi I. 2013. Exploring the bovine rumen bacterial community from birth to adulthood. Isme Journal 7(6): 1069-1079.

Joynson R, Pritchard L, Osemwekha E, Ferry N. 2017. Metagenomic analysis 504 505 506 DOI 10.1038/ismej.2013.2 of the gut microbiome of the common black slug Arion ater in search of novel lignocellulose degrading enzymes. Frontiers in Microbiolology 8:2181 DOI 10.3389/fmicb.2017.02181

Klockars J, Huffman J, Fried B. 2007. Survey of seasonal trematode infections in Helisoma trivolvis (Gastropoda) from lentic ecosystems in New Jersey, U.S.A. Comparative Parasitology 74(1): 7580 DOI 10.1654/4227.1

Kokou F, Sasson G, Friedman J, Eyal S, Ovadia O, Harpaz S, Cnaani A, 511 Mizrahi I. 2019. Core gut microbial communities are maintained by beneficial 513 interactions and strain variability in fish. Nature Microbiology 4(10): 1-10 DOI 
514 Langille M, Zaneveld J, Caporaso JG, McDonald D, Knights D, Reyes JA,

515 Clemente JC, Burkepile DE, Thurber RL, Knight R, Beiko RG,

516 Huttenhower C. 2013. Predictive functional profiling of microbial 517 communities using 16S rRNA marker gene sequences. Nature Biotechnology $518 \quad 31: 814-821$ DOI 10.1038/nbt.2676.

519 Lee KA, Kim SH, Kim EK, Ha EM, You H, Kim B, Kim MJ, Kwon Y, Ryu 520 JH, Lee WJ. 2013. Bacterial-derived uracil as a modulator of mucosal 521 immunity and gut-microbe homeostasis in Drosophila. Cell 153(4): 797-811 522 DOI 10.1016/j.cell.2013.04.009

523 Lombardo P \& Cooke GD. 2002. Consumption and preference of selected food 524 types by two freshwater gastropod species. Archiv fur Hydrobiologie 155(4): 525 667-685 DOI 10.1016/S0378-4371(02)00872-5

526

527 528 529 530

531 Design and experimental application of a novel non-degenerate universal primer 532 set that amplifies prokaryotic 16s RNA genes with a low possibility to amplify 533 eukaryotic RNA genes. DNA Research 21: 217-227 DOI 10.1093/dnares/dst052

Mikaelyan A, Meuser K, and Brune A. 2017. Microenvironmental heterogeneity of gut compartments drives bacterial community structure in wood- and humusfeeding higher termites. FEMS Microbiology Ecology 93(1):1-11 DOI

\subsection{3/femsec/fiw210}

Mori H, Maruyama F, Kato H, Toyoda A, Dozono A, and Ohtsubo Y. 2013. 
534 Muegge BD, Kuczynski J, Knights D, Clemente JC, Gonzalez A, Fontana L,

535 Henrissat B, Knight R, Gordon JI. 2011. Diet drives convergence in gut

536 microbiome functions across mammalian phylogeny and within humans.

537 Science 332(6032): 970-974 DOI 10.1126/science.1198719

538 Nicolai A, Rouland-lefèvre C, Ansart A, Filser J, Lenz R, Pando A, Charier M.

539 2015. Inter-populatIon differences and seasonal dynamic of the bacterial gut

540 community in the endangered land snail Helix pomatia (gastropoda: helicidae).

$541 \quad$ Malacologia 59(1): 177-190 DOI 10.4002/040.059.0101

542 Peterson \& Nathan A. 2007. Seasonal prevalence of Ribeiroia ondatrae in one 543 population of Planorbella trivolvis (=Helisoma trivolvis), including notes on the $544 \quad$ larval trematode component community. Comparative Parasitology 74(2): 312$545 \quad 318$ DOI $10.1654 / 4233.1$

546 Pinheiro GL, Correa RF, Cunha RS, Cardoso AM, Chaia C, Clementino MM, 547 Garcia ES, de Souza W, and Frasés S. 2015. Isolation of aerobic cultivable 548 cellulolytic bacteria from different regions of the gastrointestinal tract of giant 549 land snail Achatina fulica. Frontiers in Microbiology 6:860 DOI $550 \quad 10.3389 /$ fmicb.2015.00860

551 Qi W, Nong G, Preston JF, Ben-Ami F, Ebert D. 2009. Comparative 552 metagenomics of Daphnia symbionts. BMC Genomics 10(1): 172 DOI 553 $10.1186 / 1471-2164-10-172$ 
554 Russell JA, Moreau CS, Goldman-Huertas B, Fujiwara M, Lohman DJ,

555 Pierce NE. 2009. Bacterial gut symbionts are tightly linked with the evolution

556 of herbivory in ants. Proceedings of the National Academy of Sciences of the

557 United States of America 106: 21236-21241 DOI 10.1073/pnas.0907926106

558 Schloss PD, Gevers D, Westcott SL. 2011. Reducing the effects of PCR 559 amplification and sequencing artifacts on 16S rRNA-based studies. PLoS One 6(12): e27310 DOI 10.1371/journal.pone.0027310

561 Sharma R, Schumacher U, Ronaasen V, Coates M. 1995. Rat intestinal mucosal 562 responses to a microbial flora and different diets. Gut 36: 209-214 DOI 563 10.1136/gut.36.2.209

564 Shade A, \& Handelsman J. 2012. Beyond the venn diagram: the hunt for a core 565 microbiome. Environmental Microbiology 14(1): 4-12 DOI 10.1111/j.1462$566 \quad 2920.2011 .02585 . x$

567 Speiser, B, 2001. Behaviour, feeding, food and feeding behaviour. Biology of 568 Terrestrial Molluscs 112(1): 21-35 DOI 10.1079/9780851993188.0259

569 Segata N, Izard J, Waldron L, Gevers D, Miropolsky L, Garrett WS, 570 Huttenhower C. 2011. Metagenomic biomarker discovery and explanation. 571 Genome Biology 12:R60 DOI 10.1186/gb-2011-12-6-r60 
572 Stark A, Nyska A, Madar Z. 1996. Metabolic and morphometric changes in

573 small and large intestine in rats fed high-fiber diets. Toxicologic Pathology

574

24(2): 166-171 DOI 10.1177/019262339602400204

575 Tun HM, Mauroo NF, Yuen CS, Ho JCW, Wong MT, Leung CC. 2014.

576 Microbial diversity and evidence of novel homoacetogens in the gut of both

577 geriatric and adult giant pandas (ailuropoda melanoleuca). Plos One 9(1):

578 e79902 DOI 10.1371/journal.pone.0079902

579

580

581

582

583

584

585

586

587

588

589

590

591

Van Horn DJ, Garcia JR, Loker ES, Mitchell KR, Mkoji GM, Adema CM, Takacs-Vesbach CD. 2012. Complex intestinal bacterial communities in three species of planorbid snails. Journal of Molluscan Studies 78(1):74-80 DOI 10.1093/mollus/eyr038

Vesbach CT, King K, Van Horn D, Larkin K, Neiman M. 2016. Distinct bacterial microbiomes in sexual and asexual potamopyrgus antipodarum, a New Zealand freshwater snail. PLOS ONE 11(8): e0161050. DOI 10.1371/journal.pone.0161050

Wijanarka W, Kusdiyantini E, Parman S. 2016. Screening cellulolytic bacteria from the digestive tract snail (Achatina fulica) and test the ability of cellulase activity. Biosaintifika 8 (3): 385-391 DOI 10.15294/biosaintifika.v8i3.7263

Zhao T, Yang SB, Chen G.H, Xu YH, Xu YC, Luo Z, 2020. Dietary glucose increases glucose absorption and lipid deposition via SGLT1/2 signaling and 
592 acetylated ChREBP in the intestine and isolated intestinal epithelial cells of 593 yellow catfish. The Journal of Nutrition 150:1790-1798 DOI $594 \quad 10.1093 /$ jn/nxaa125

595

596 
Figure 1

Figure 1 Effects of different diets on intestinal histology of Planorbella trivolvis.

A: Intestinal morphology of HV snails $(200 \mu \mathrm{m})$; B: Intestinal morphology of HV snails (50 $\mu \mathrm{m}) ; \mathrm{C}$ : Intestinal morphology of NHV snails $(200 \mu \mathrm{m})$; D: Intestinal morphology of NHV snails $(50 \mu \mathrm{m})$.
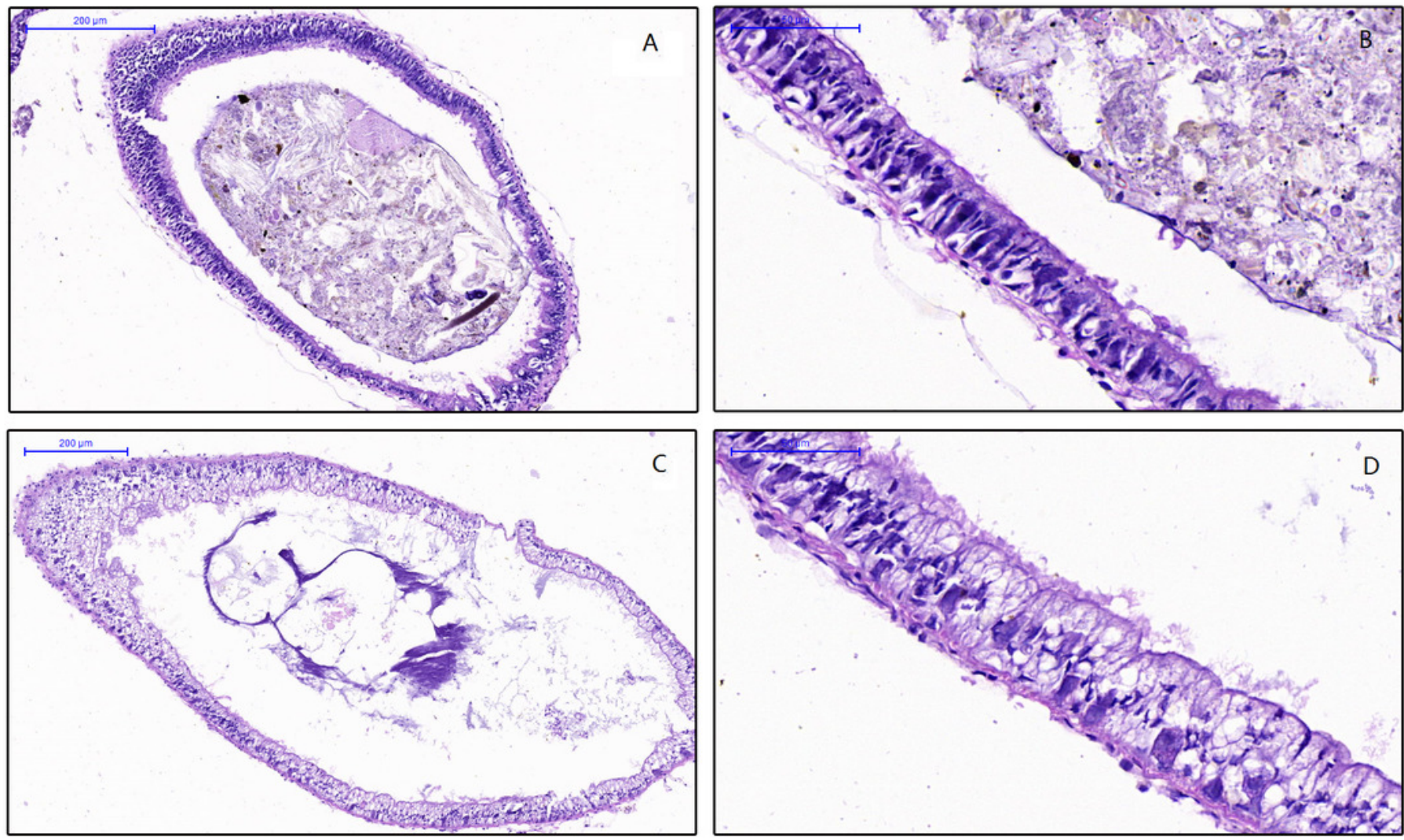


\section{Figure 2}

Figure 2 Diversity of gut community in snails fed with diiferent diets types.

A-C: Rarefaction curves (A), Rank-abundance curves (B), and core analysis (C) of intestinal microbial flora at OTU level; D-F: VENN analysis of gut community at phylum (D), family (E), and genus (F) level.

A

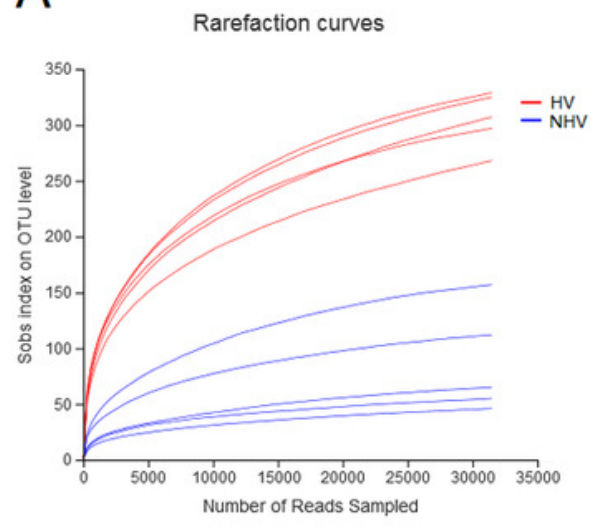

D

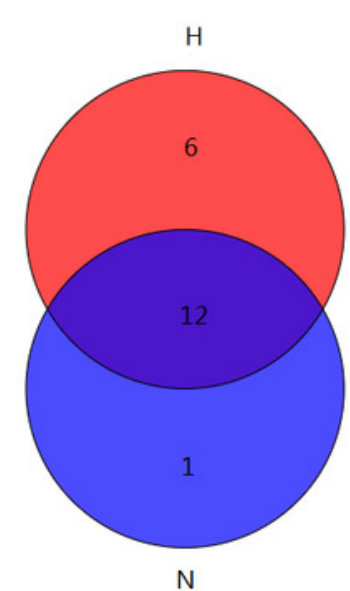

B

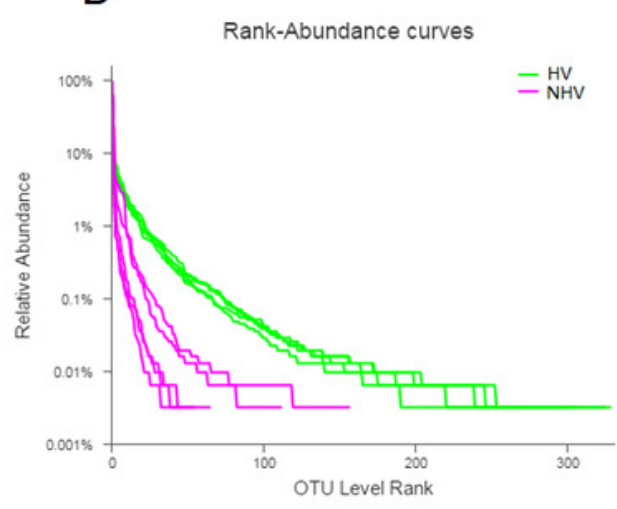

$E$

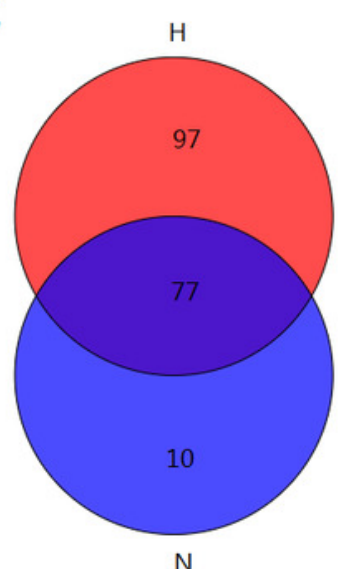

C

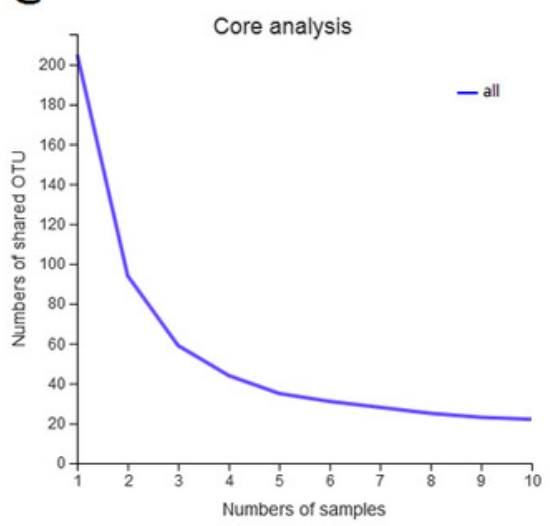

F

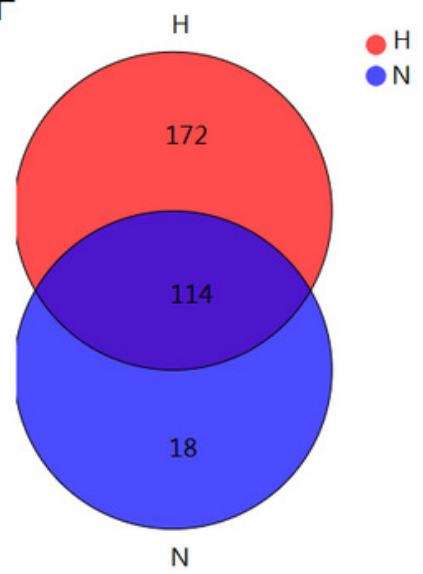


Figure 3

Figure 3 Composition of intestinal microbial flora at phylum(A), family (B) and genus (C) level.

The bacterial taxon with abundance below $1 \%$ in phylum, $4 \%$ in family, and $3 \%$ in genus level was merged and shows as "others". 

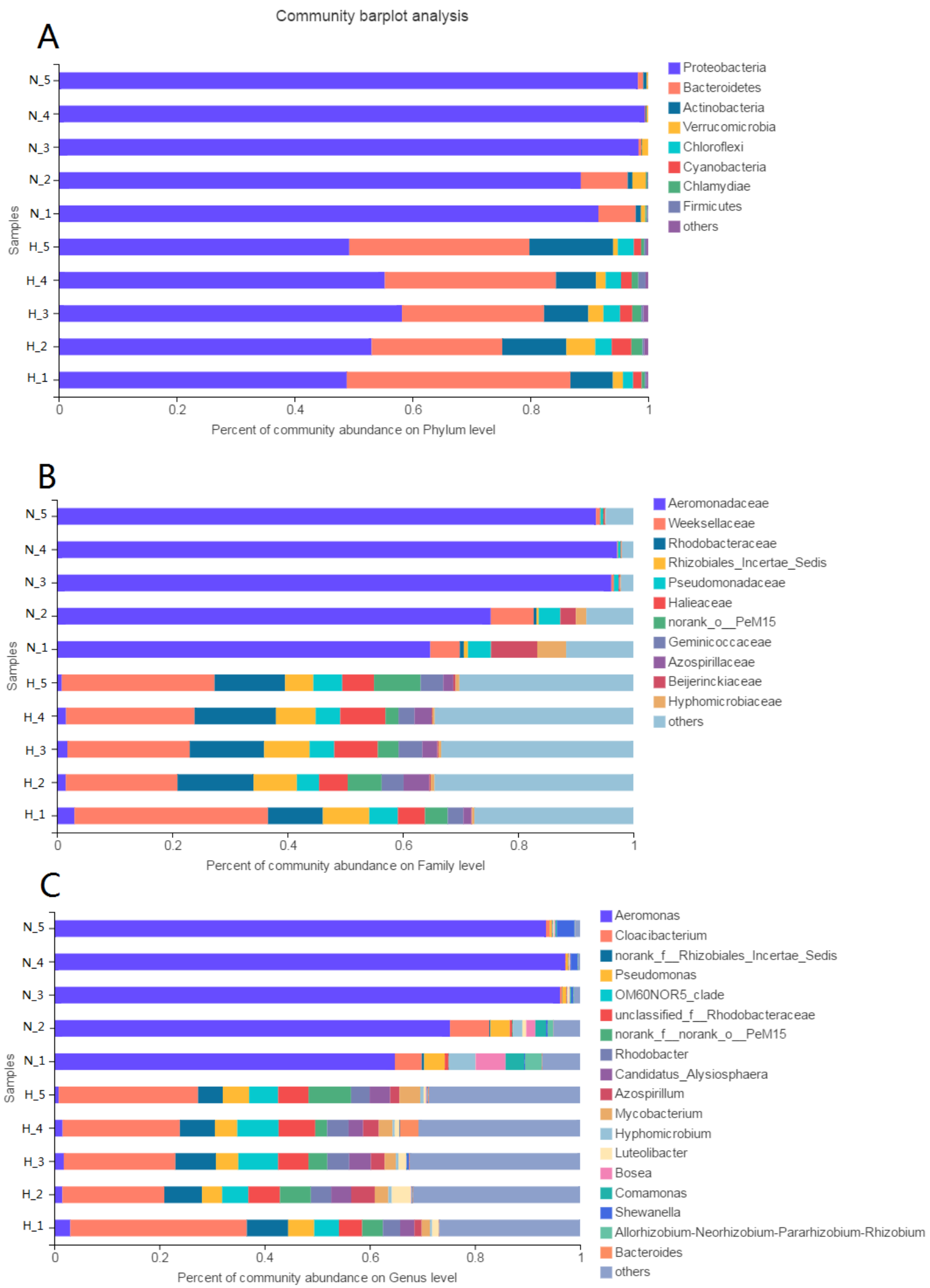


\section{Figure 4}

Figure 4 Heat map showing the relative abundance of the 35 bacterial genus in snail.

The HV snails are shown by $\mathrm{H1}, \mathrm{H2}, \mathrm{H} 3, \mathrm{H} 4, \mathrm{H} 5$ and NHV snails are shown by N1, N2, N3, A4, N5. The red asterisk represented the significantly different genera among two groups, based on wilcoxon rank-sum test, “*” represent $\mathrm{P}<0.05$, “**" "represent $\mathrm{P}<0.01$. 


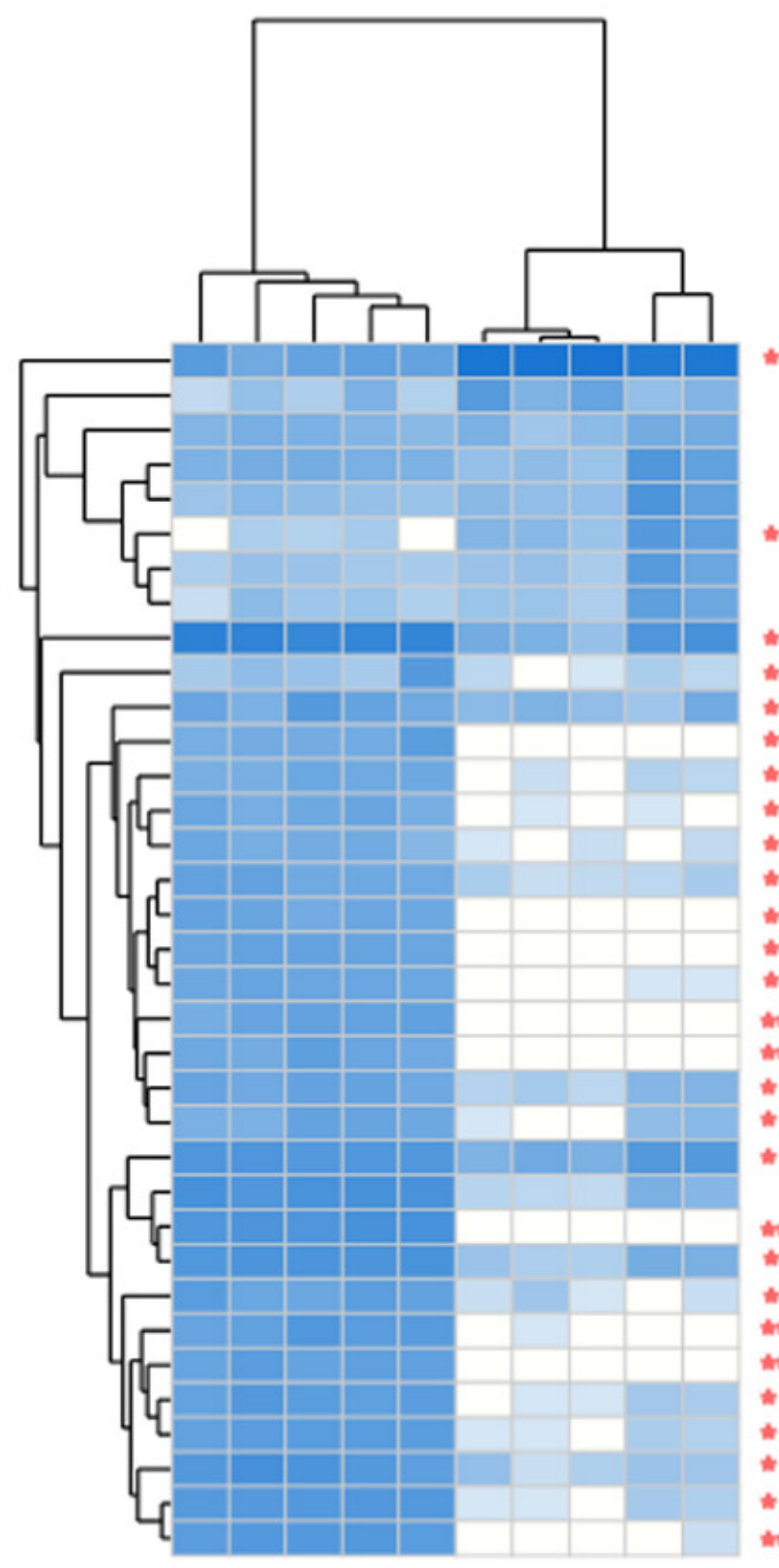

Aeromonas (2.8\%//85.4\%)

Shewanella $(0.1 \% / 2.15)$

unclassified_f_Demequinaceae (0.3\%/0.4\%)

Hyphomicrobium (0.55/2.45)

Bosea (0.1\%/2.6\%)

* Comamonas (0.02\%:/2.2\%)

Allorhizobium-Neorhizobium-Pararhizobium-Rhizobium (0.05\%/0.995) unclassified_f_Beijerinckiaceae (0.07\%/0.75)

Cloacibacterium (24.6\%/2.85)

Bacteroides (0.5\%/0.01\%)

Luteolibacter (2.5\%/0.35)

th Uliginosibacterium (20\%/0\%5)

norank_f_norank_o_Chloroplast (0.59//0.019)

Delftia (0.95/0.001\%)

Sandaracinobacter (0.79/0.0045)

Flavobacterium (2.25/0.025)

Dinghuibacter (1.19//0\%)

Thioclava (2.25\%/0\%5)

Roseomonas (2.195/0.0015)

Roseobacter_clade_CHAB-1-5_lineage (2.4\%/05)

norank_f_norank_o norank_c_Sericytochromatia (2.29\%/095)

Reyranella (1.25\%/0.15)

unclassified_f__Parachlamydiaceae (205\%/0.07\%)

Pseudomonas (4.5\%/2.5\%)

norank_f_Rhizobiales_Incertae_Sedis (6.5\%/0.19\%)

OMOONOR5_clade (6.19\%/O95)

unclassified_f_Rhodobacteraceae (5.5\%/0.25)

unclassified_f_Enterobacteriaceae (1.9\%//0.015)

Azospirillum (2.65\%/0.0015\%)

Polymorphobacter (1.9\%/095)

Mycobacterium (2.6\%/0.025)

norank_f_JG30-KF-CM45 (2.5\%/0.019)

norank_f_norank_o_PeM15 (4.55/0.05\%)

Rhodobacter (3.5\%/,0.025)

Wh Candidatus_Alysiosphaera (3.5\%/0.001\%)

$\begin{array}{llllllllllllllllllllllll}\mathrm{H} 1 & \mathrm{H} 2 & \mathrm{H} 3 & \mathrm{H} 4 & \mathrm{H} 5 & \mathrm{~N} 1 & \mathrm{~N} 2 & \mathrm{~N} 3 & \mathrm{~N} 4 & \mathrm{~N} 5\end{array}$ 
Figure 5

Figure 5 Principal Coordinates Analysis (PCOA) plots in intestinal microbiota of snails at OTU level using pairwise unweighted (A) and weighted UniFrac distances (B).

The HV snails are shown by blue dot and NHV snails are shown by orange triangle. Adonis: $\mathrm{p}$ value $=0.004, \mathrm{R}$-value $=0.4916(\mathrm{~A}) ; \mathrm{p}$-value $=0.004, \mathrm{R}$-value $=0.9170(\mathrm{~B})$.

A

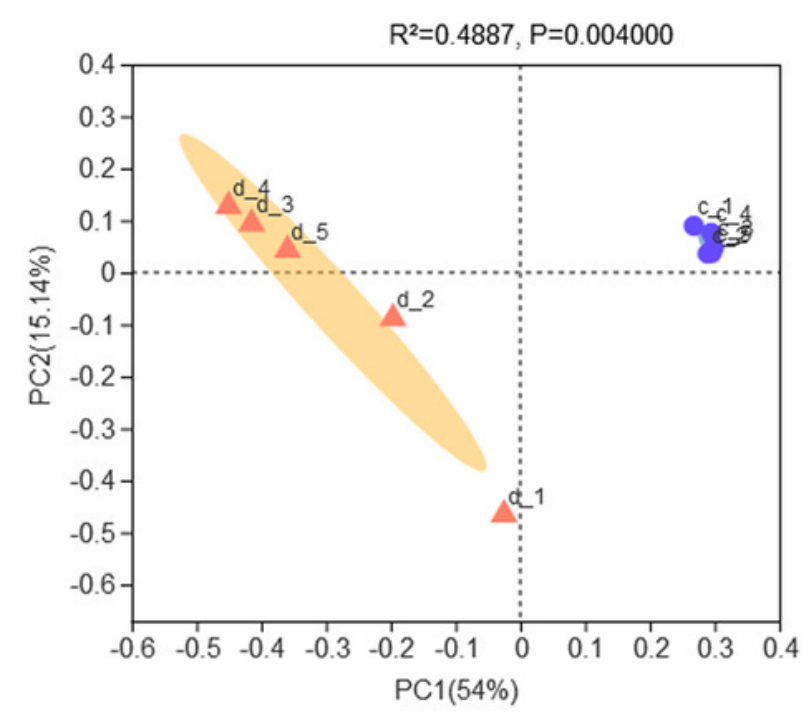

B

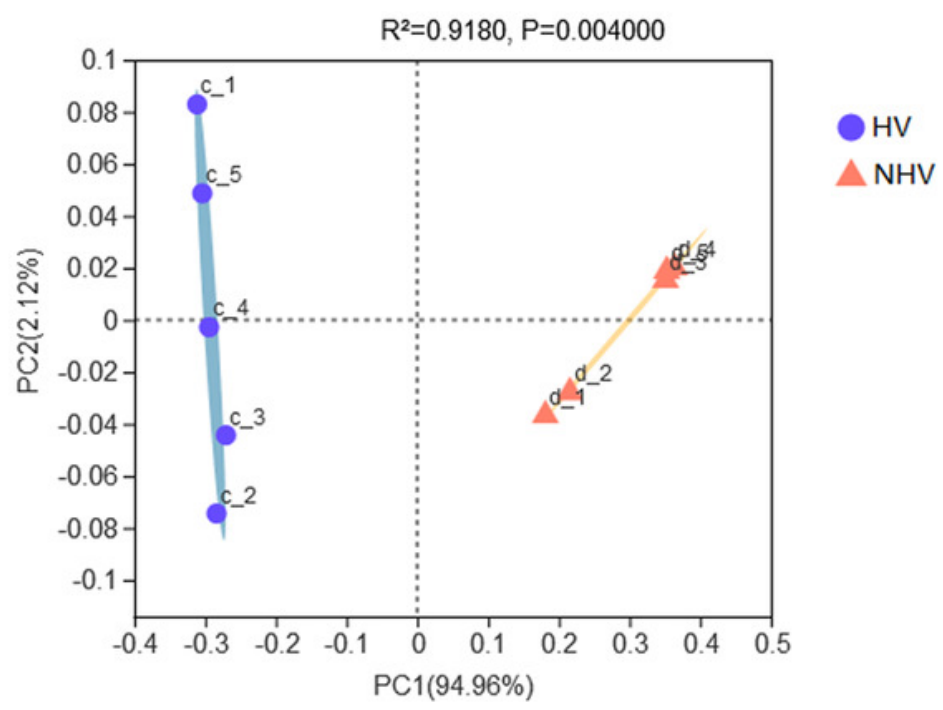




\title{
Figure 6
}

Figure 6 Predictive functional profiles generated from 16S rRNA gene sequences using PICRUSt.

\section{Significant differences between HV and NHV snails were observed for several COG function (} two-tailed Student's t-test ; $* \mathrm{P}<0.05, * * \mathrm{P}<0.01)$. The HV group is represented by green bars (H) and the NHV group by red bars (N).

\author{
Cytoskeleton \\ Extracellular structures \\ Defense mechanisms
}

Intracellular trafficking, secretion, and vesicular transport

Signal transduction mechanisms

Function unknown

General function prediction only

Secondary metabolites biosynthesis, transport and catabolism

Inorganic ion transport and metabolism

Posttranslational modification, protein turnover, chaperones

Cell motility

Cell wall/membrane/envelope biogenesis

Replication, recombination and repair

Transcription

Translation, ribosomal structure and biogenesis

Lipid transport and metabolism

Coenzyme transport and metabolism

Carbohydrate transport and metabolism

Nucleotide transport and metabolism

Amino acid transport and metabolism

Cell cycle control, cell division, chromosome partitioning

Energy production and conversion

Chromatin structure and dynamics

RNA processing and modification

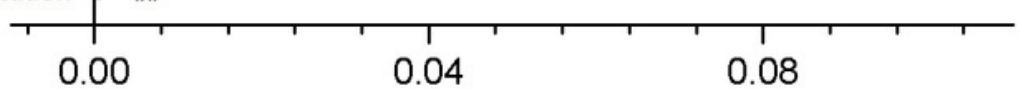

COG function classification

Relative abundance 


\section{Figure 7}

Figure 7 Predictive KEGG Orthology (KO) of lignocellulose-active enzymes across diet types.

$\mathrm{H} 1$ to $\mathrm{H} 5$ (red color) indicates the sequence number of predicted genes among the HV snails, and $\mathrm{N} 1$ to $\mathrm{N} 5$ indicates the sequence number of predicted genes among the NHV snails. Each column represents a different entry in the greengene database, each with a unique K-number 

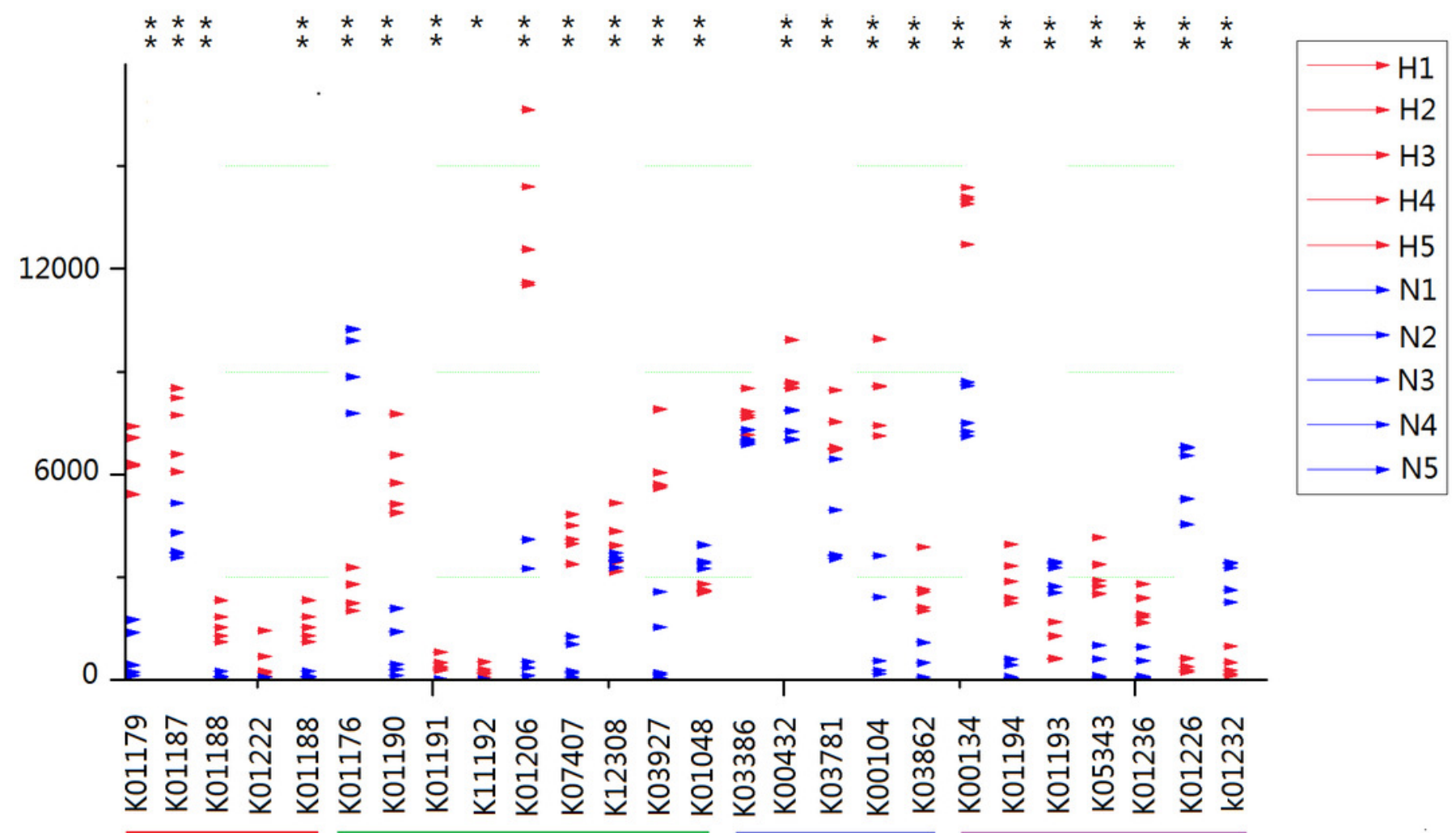

Cellulose

Hemicellulose

Lignin

Cello-oligosaccharides

K01179: endoglucanase [EC:3.2.1.4]; K01187: alpha-glucosidase [EC:3.2.1.20]; K01188: beta-glucosidase [EC:3.2.1.21]; K01222: 6-phospho-beta-glucosidase [EC:3.2.1.86]; K01188: beta-glucosidase [EC:3.2.1.21]; K01176: alpha-amylase [EC:3.2.1.1]; K01190: beta-galactosidase [EC:3.2.1.23]; K01191: alphamannosidase [EC:3.2.1.24]; K11192: PTS system, N-acetylmuramic acid-specific IIC component; K01206: alpha-L-fucosidase [EC:3.2.1.51]; K07407: alphagalactosidase [EC:3.2.1.22]; K12308: beta-galactosidase [EC:3.2.1.23]; K03927: carboxylesterase 2 [EC:3.1.1.1]; K01048: lysophospholipase [EC:3.1.1.5]; K03386: peroxiredoxin (alkyl hydroperoxide reductase subunit C) [EC:1.11.1.15]; K00432: glutathione peroxidase [EC:1.11.1.9]; K03781: catalase [EC:1.11.1.6]; K00104: glycolate oxidase [EC:1.1.3.15]; K03862: vanillate monooxygenase [EC:1.14.13.82]; K00134: glyceraldehyde 3-phosphate dehydrogenase [EC:1.2.1.12]; K01194: alpha,alpha-trehalase [EC:3.2.1.28]; K01193: beta-fructofuranosidase [EC:3.2.1.26]; K05343: maltose alpha-D-glucosyltransferase / alpha-amylase [EC:5.4.99.16/3.2.1.1]; K01236: maltooligosyltrehalose trehalohydrolase [EC:3.2.1.141]; K01226: trehalose-6-phosphate hydrolase [EC:3.2.1.93]; K01232: maltose-6'-phosphate glucosidase [EC:3.2.1.122]. 
Figure 8

Predictive KEGG Orthology $(\mathrm{KO})$ of enzymes related to protein degradation in snails.

$\mathrm{H} 1$ to $\mathrm{H} 5$ (red color) indicates the sequence number of predicted genes among the HV snails, and $\mathrm{N} 1$ to $\mathrm{N} 5$ indicates the sequence number of predicted genes among the NHV snails. Each column represents a different entry in the greengene database, each with a unique Knumber. The differences of each KO genes were showed by "*" at the top of plot. "*" represent $\mathrm{P}<0.05$, “**"” "represent $\mathrm{P}<0.01$. 


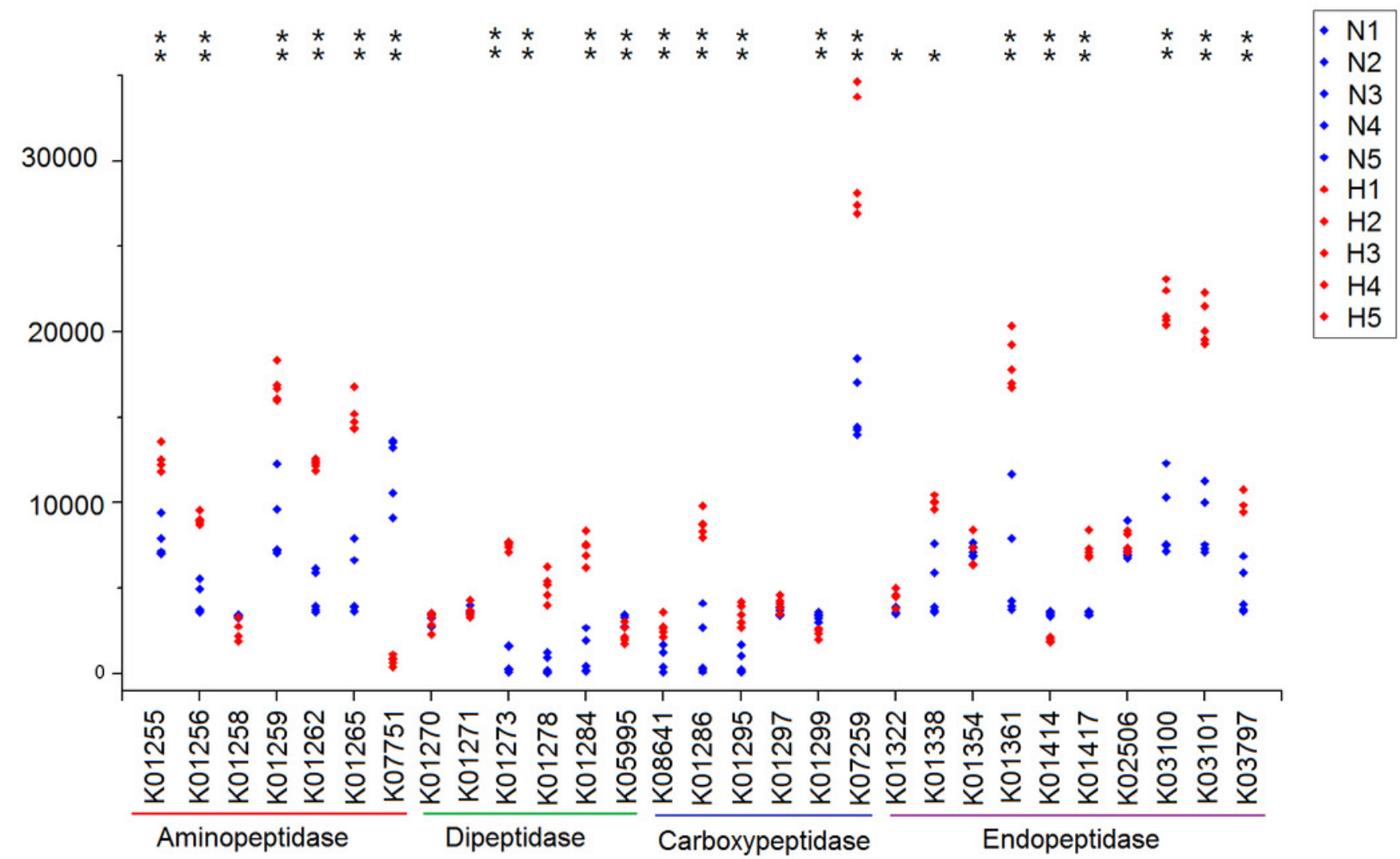

K01255: leucyl aminopeptidase [EC:3.4.11.1]; K01256: aminopeptidase N [EC:3.4.11.2]; K01258: tripeptide aminopeptidase [EC:3.4.11.4]; K01259: proline iminopeptidase [EC:3.4.11.5]; K01262: Xaa-Pro aminopeptidase [EC:3.4.11.9]; K01265: methionyl aminopeptidase [EC:3.4.11.18]; K07751: PepB aminopeptidase [EC:3.4.11.23]; K01270: dipeptidase D [EC:3.4.13.-]; K01271: Xaa-Pro dipeptidase [EC:3.4.13.9]; K01273: membrane dipeptidase [EC:3.4.13.19]; K01278: dipeptidyl-peptidase 4 [EC:3.4.14.5]; K01284: peptidyl-dipeptidase Dcp [EC:3.4.15.5]; k05995: dipeptidase E [EC:3.4.13.21]; k08641: D-alanyl-D-alanine dipeptidase [EC:3.4.13.22]; K01286: D-alanyl-D-alanine carboxypeptidase [EC:3.4.16.4]; K01295: glutamate carboxypeptidase [EC:3.4.17.11]; K01297: muramoyltetrapeptide carboxypeptidase [EC:3.4.17.13]; K01299: carboxypeptidase Taq [EC:3.4.17.19]; k07259: D-alanyl-D-alanine carboxypeptidase [EC:3.4.16.4]; K01322: proly oligopeptidase [EC:3.4.21.26]; K01338: ATP-dependent Lon protease [EC:3.4.21.53]; K01354: oligopeptidase B [EC:3.4.21.83]; K01361: lactocepin [EC:3.4.21.96]; K01414: oligopeptidase A [EC:3.4.24.70]; K01417: extracellular elastinolytic metalloproteinase [EC:3.4.24.-]; k02506: leader peptidase HopD [EC:3.4.23.43]; k03100: signal peptidase I [EC:3.4.21.89]; k03101: signal peptidase II [EC:3.4.23.36]; k03797: carboxyl-terminal processing protease [EC:3.4.21.102] 


\section{Table $\mathbf{1}$ (on next page)}

Table 1 Chemical characteristics of alfalfa $(\mathrm{g} / 100 \mathrm{~g})$.

HV (alfalfa): the herbivorous snails reared with alflafa; NHV(pellet feed): the non-herbivorous snails reared with pellet feed. The data on the composition of the pellet feed come from the manufacturer (Takara sakana-ii). The composition of the alfalfa was determined in laboratory as follwing methods: (1)The NDF and ADF levels were measured as described by Van Soest et al. (1991). (2) The crude protein (CP) content was measured by the Kjeldahl N method (AOAC 2000). (3) Soxhlet immersion extraction was used for measure crude fat. (4) Crude ash was measured by firing the sample for $30 \mathrm{~min}$ under $550{ }^{\circ} \mathrm{C}$ in muffle furnace. 
1

\begin{tabular}{lllll}
\hline Diets type & Protein & Crude fiber & Crude fat & Crude ash \\
\hline HV(alfalfa) & $19.62 \pm 0.23$ & $26.75 \pm 0.24$ & $4.86 \pm 0.11$ & $8.34 \pm 0.02$ \\
NHV(pellet feed) & $27.22 \pm 0.09$ & $4.97 \pm 0.03$ & $2.17 \pm 0.05$ & $9.03 \pm 0.06$ \\
\hline
\end{tabular}

2

3

4

5

6 


\section{Table 2 (on next page)}

Table 2 Growth performance of Planorbella trivolvis.

In the same column, values with no letter or the same letter superscripts mean no significant difference $(P>0.05)$, while with different letter superscripts mean significant difference $(P<$ 0.05). HV, the herbivorous snails reared with alflafa; NHV, the non-herbivorous snails reared with pellet feed. SGR: special growth rate; WGR: Weight gain rate. 
1

\begin{tabular}{|c|c|c|c|c|c|}
\hline Items & Groups & Initial value & difference & Final value & difference \\
\hline \multirow[t]{2}{*}{$\begin{array}{l}\text { diameter of } \\
\text { shell/mm }\end{array}$} & HV & $5.98 \pm 0.07$ & $\mathrm{a}$ & $10.53 \pm 0.45$ & b \\
\hline & NHV & $5.78 \pm 0.13$ & $\mathrm{a}$ & $11.86 \pm 0.11$ & $\mathrm{a}$ \\
\hline \multirow[t]{2}{*}{ Weight/g } & $\mathrm{HV}$ & $0.068 \pm 0.003$ & $\mathrm{a}$ & $0.219 \pm 0.003$ & $\mathrm{~b}$ \\
\hline & NHV & $0.065 \pm 0.005$ & $\mathrm{a}$ & $0.432 \pm 0.007$ & $\mathrm{a}$ \\
\hline \multirow[t]{2}{*}{ WGR/\% } & $\mathrm{HV}$ & 1 & & $321.40 \pm 16.01$ & $\mathrm{~b}$ \\
\hline & NHV & 1 & & $566.90 \pm 46.28$ & $\mathrm{a}$ \\
\hline \multirow[t]{2}{*}{$\mathrm{SGR} / \%$} & $\mathrm{HV}$ & 1 & & $1.94 \pm 0.08$ & $\mathrm{~b}$ \\
\hline & NHV & 1 & & $3.16 \pm 0.11$ & $\mathrm{a}$ \\
\hline
\end{tabular}

2

3 


\section{Table 3(on next page)}

Table 3 alpha-diversity indices of intestinal microbial flora of Planorbella trivolvis.

$\mathrm{HV}$, the herbivorous snails reared with alflafa; NHV, the non-herbivorous snails rearedwith pellet feed. $P$-values were from a t-test. 
1

\begin{tabular}{lccccc}
\hline Estimators & HV-Mean & HV-Sd & NHV-Mean & NHV-Sd & P-value \\
\hline OUT number & 307.60 & 25.71 & 90.00 & 46.67 & 0 \\
Sequences number & 54277.60 & 6298.01 & 39606.6 & 6846.06 & 0.008 \\
sobs & 305.2 & 24.56 & 87.00 & 46.67 & $1.513 \mathrm{e}-05$ \\
shannon & 3.42 & 0.19 & 1.00 & 0.50 & $7.253 \mathrm{e}-06$ \\
simpson & 0.09 & 0.03 & 0.61 & 0.17 & 0.000133 \\
ace & 379.08 & 29.34 & 118.69 & 47.56 & $6.243 \mathrm{e}-06$ \\
chao & 375.04 & 26.28 & 105.4 & 47.40 & $3.809 \mathrm{e}-06$ \\
coverage & 0.9976 & 0.0003 & 0.9992 & 0.0003 & $4.689 \mathrm{e}-05$ \\
\hline
\end{tabular}

2

3

4

5

6

7 


\section{Table 4(on next page)}

Table 4 Shared core bacteria among two groups.

HV, the herbivorous snails reared with alflafa; NHV, the non-herbivorous snails reared with pellet feed. 
2

\begin{tabular}{|c|c|c|c|c|c|c|}
\hline Phylum & Family & Genus & $\mathrm{HV}$ & SD & NHV & $\mathrm{SD}$ \\
\hline Proteobacteria & Rhizobiaceae & Allorhizobium & 0.07 & 0.03 & 0.91 & 1.38 \\
\hline Bacteroidetes & Flavobacteriaceae & Flavobacterium & 1.17 & 0.45 & 0.02 & 0.01 \\
\hline Proteobacteria & Rhizobiaceae & norank & 0.01 & 0.01 & 0.12 & 0.17 \\
\hline Proteobacteria & Shewanellaceae & Shewanella & 0.11 & 0.16 & 1.11 & 1.36 \\
\hline Proteobacteria & Rhodobacteraceae & Rhodobacter & 3.78 & 0.37 & 0.02 & 0.02 \\
\hline Bacteroidetes & Weeksellaceae & Cloacibacterium & 24.6 & 5.66 & 2.75 & 3.33 \\
\hline Proteobacteria & Aeromonadaceae & Aeromonas & 1.78 & 0.8 & 85.4 & 14.57 \\
\hline Verrucomicrobia & Rubritaleaceae & Luteolibacter & 1.53 & 1.25 & 0.3 & 0.27 \\
\hline Proteobacteria & Reyranellaceae & Reyranella & 1.21 & 0.34 & 0.13 & 0.15 \\
\hline Bacteroidetes & Cytophagaceae & Cytophaga & 0.07 & 0.07 & 0.07 & 0.08 \\
\hline Proteobacteria & Sphingomonadaceae & Altererythrobacter & 0.02 & 0.01 & 0.02 & 0.02 \\
\hline Proteobacteria & Rhizobiales_Incertae_Sedis & unclassified & 0.15 & 0.03 & 0.06 & 0.07 \\
\hline Verrucomicrobia & Rubritaleaceae & Haloferula & 0.05 & 0.05 & 0.6 & 0.59 \\
\hline Bacteroidetes & Chitinophagaceae & Sediminibacterium & 0.11 & 0.05 & 0.26 & 0.37 \\
\hline Actinobacteria & Demequinaceae & unclassified & 0.35 & 0.13 & 0.41 & 0.29 \\
\hline Actinobacteria & Mycobacteriaceae & Mycobacterium & 2.58 & 0.88 & 0.02 & 0.02 \\
\hline Proteobacteria & Beijerinckiaceae & unclassified & 0.07 & 0.07 & 0.68 & 0.98 \\
\hline Proteobacteria & Xanthomonadaceae & Stenotrophomonas & 0.06 & 0.02 & 0.05 & 0.08 \\
\hline Proteobacteria & Enterobacteriaceae & Kluyvera & 0.56 & 0.58 & 0.02 & 0.04 \\
\hline Proteobacteria & Pseudomonadaceae & Pseudomonas & 4.47 & 0.5 & 1.84 & 1.81 \\
\hline Proteobacteria & Enterobacteriaceae & unclassified & 1.85 & 0.82 & 0.02 & 0.03 \\
\hline Actinobacteria & norank_o_PeM15 & norank & 4.75 & 2.27 & 0.06 & 0.05 \\
\hline Chloroflexi & JG30-KF-CM45 & norank & 2.51 & 0.45 & 0.01 & 0.01 \\
\hline Proteobacteria & Rhodobacteraceae & unclassified & 5.79 & 0.91 & 0.25 & 0.28 \\
\hline Proteobacteria & Rhizobiales_Incertae_Sedis & norank & 6.81 & 1.27 & 0.15 & 0.2 \\
\hline Proteobacteria & Beijerinckiaceae & Bosea & 0.12 & 0.06 & 1.58 & 2.41 \\
\hline Proteobacteria & Hyphomicrobiaceae & Hyphomicrobium & 0.51 & 0.12 & 1.42 & 2.12 \\
\hline
\end{tabular}

\section{3}

4

5

6 